\title{
A New Era of Endoscopic Ultrasound-Guided Tissue Acquisition for Next-Generation Sequencing for Pancreatic Cancer
}

\author{
Tae Jun Song \\ Division of Gastroenterology, Asan Medical Center, University of Ulsan College of Medicine, Seoul, Korea
}

\author{
See "Factors of Endoscopic Ultrasound-Guided Tissue Acquisition for Successful Next-Generation Sequencing in Pancreatic \\ Ductal Adenocarcinoma" by Jae Keun Park, et al. on page 387, Vol. 14, No. 3, 2020
}

Over the past decade, genomics and bioinformatics have markedly evolved and has allowed the identification of numerous biomarkers of many malignancies. ${ }^{1}$ Especially, the development of next-generation sequencing (NGS) has enabled the fast and precise identification of various mutations. Genomic data generated by NGS might eventually lead to the identification of novel biomarkers that can be used to screen for malignancy and to develop a new treatment. ${ }^{1}$ The role of NGS for both the development of new treatments and precision medicine is getting more and more attention. ${ }^{2}$ Precision medicine refers to the tailoring of therapy based on an individual patient's genetics, lifestyle, and environment. Characterizing genomic mutation in tumors for predictive and prognostic purposes by NGS has become an essential part of precision medicine. The NGS can provide a comprehensive view of an individual malignancy, which can help real-time clinical decision-making.

In patients with pancreatic cancer $(\mathrm{PCa})$, precision medicine has not been well established. Notably, the possible reason for the poor prognosis of PCa is the lack of ideal biomarkers for early diagnosis and therapeutic stratification. Therefore, NGS was expected to provide a new turning point in the diagnosis and treatment of PCa. Recently, the studies on the role of NGS in the diagnosis of PCa have been rapidly growing. However, NGS for PCa is still in its infancy. Many potential targets have been identified, but no definitive biomarker was developed yet.

Endoscopic ultrasound-guided tissue acquisition (EUS-TA) is the primary method to obtain tissue samples from $\mathrm{PCa}{ }^{2} \mathrm{Al}-$ though EUS-TA has already been proven to be highly accurate in the diagnosis of PCa, inconclusive results are not uncommon in cytopathologic diagnosis. NGS may enable the sequencing of multiple genes in a limited number of samples obtained by EUS-TA for PCa and identification of potential mutations as diagnostic and therapeutic targets.

About $90 \%$ of PCa are pancreatic ductal adenocarcinomas (PDAC), and the KRAS mutation has been found in over 95\% of PDAC. ${ }^{3}$ So, many studies validated NGS using KRAS mutation as a reference gene. Until now, there has been no prospective study evaluating the success rate of NGS for PCa using EUSTA samples. Several retrospective studies have reported that the success rates of NGS using EUS-TA samples are between 60\% and 100\%. ${ }^{4}$ Kameta et al. ${ }^{5}$ showed that KRAS mutations were detected in 26 of 27 PDAC (96\%) and none of the 11 non-PDAC by NGS using EUS-TA samples. De Biase et al. ${ }^{6}$ founded out the KRAS mutations of 60 EUS-guided fine needle aspiration (EUSFNA) samples and compared the results of Sanger sequencing, NGS, and allele specific locked nucleic acid quantitative polymerase chain reaction (PCR). They reported that the sensitivity for detecting the KRAS mutation was higher in NGS (73.7\%) compared with those in Sanger sequencing (42.1\%) or in allele specific locked nucleic acid quantitative PCR (52.8\%).

Several factors might influence the successful NGS for PCa using EUS-TA.

For successful NGS, cellularity and tumor fraction in EUSTA samples are essential. Samples with low cellularity and low tumor fraction have an increased risk of unsuccessful NGS. ${ }^{2}$ Generally, NGS requires a tumor fraction of $\geq 20 \%$. Since PCa also contains stromal cells, hematopoietic cells and desmoplastic fibroblasts besides tumor cells, the NGS may be unsuccessful due to the possible contamination of these non-tumor cells. Although EUS-TA shows high diagnostic accuracy, the total

\footnotetext{
Correspondence to: Tae Jun Song

Division of Gastroenterology, Asan Medical Center, University of Ulsan College of Medicine, 88 Olympic-ro 43-gil, Songpa-gu, Seoul 05505, Korea

Tel: +82-2-3010-3180, Fax: +82-2-485-5782, E-mail: drsong@amc.seoul.kr pISSN 1976-2283 eISSN 2005-1212 https://doi.org/10.5009/gnl20130

(c) This is an Open Access article distributed under the terms of the Creative Commons Attribution Non-Commercial License (http://creativecommons.org/licenses/by-nc/4.0) which permits unrestricted non-commercial use, distribution, and reproduction in any medium, provided the original work is properly cited.
} 
amount of the obtained sample may be limited. Therefore, the acquisition of a large amount of samples with good quality is an integral part of NGS using EUS-TA.

The most crucial issue regarding sampling for NGS may be an adequate selection of EUS-TA methods between EUS-FNA and EUS-guided fine needle biopsy (FNB). Theoretically, the EUS-FNB needle can obtain more samples and may be optimal for NGS. Several studies reported that the success rate of NGS was higher when using EUS-FNB. Larson et al. ${ }^{7}$ evaluated the success rate of NGS using $61 \mathrm{PCa}$ samples, which were obtained by EUS-FNA $(n=7)$ or EUS-FNB $(n=54)$. They reported the success rate of NGS using EUS-FNB (70.4\%) was higher than that of EUS-FNA (42.9\%). Elhanafi et al. ${ }^{8}$ evaluated the adequacy of 167 EUS-TA samples of PCa for NGS. The success rate for NGS using EUS-FNB samples (90.9\%) was higher than that using EUS-FNA (66.9\%; p<0.05). This study showed EUS-FNB could provide enough samples for NGS compared with EUS-FNA, especially in tumors $\leq 3 \mathrm{~cm}$. However, the available literature comparing EUS-FNA and EUS-FNB are from the retrospective studies with limited numbers of samples, requiring further prospective study.

At present, different sizes of needles for EUS-TA are available from 19- to 25-gauge needles. Theoretically, larger needles can obtain a more significant amount of samples. However, there is no increase in diagnostic yield with larger needles compared with smaller needles. ${ }^{9}$ This result may be due to that larger needles may have more blood contamination compared with smaller needles, which may affect cytological diagnosis. Since large amounts of DNA or RNA are required for successful NGS, a larger needle may have a beneficial effect rather than a smaller needle for NGS.

Park et al. ${ }^{10}$ performed this study to find out the optimal approach for NGS using EUS-TA. In this study, the yield of NGS for PCa using EUS-TA samples was 57.4\%. They showed that the needle size for EUS-TA was an independent factor associated with successful NGS by multivariate analysis. It is significant in that this study showed EUS-TA with larger needles has advantages in NGS for PCa. However, because of the retrospective nature of this study, they used remaining fresh frozen samples, which were collected without intention for NGS after a clinical diagnostic examination. As a result, some of the patients had a too-small amount of DNA for NGS. DNA amounts of remaining samples obtained by 25-gauge needle were significantly lower than those obtained by 19- or 22-gauge needles. Thus, further prospective study using recently developed new EUS-FNB needles, which potentially increase tissue core procurement and reduce blood contamination is expected.

The advances in NGS for PCa using EUS-TA samples will eventually improve the early diagnosis and treatment outcomes of this most lethal disease.

\section{CONFLICTS OF INTEREST}

No potential conflict of interest relevant to this article was reported.

\section{ORCID}

Tae Jun Song

https://orcid.org/0000-0002-6156-8746

\section{REFERENCES}

1. Shen GQ, Aleassa EM, Walsh RM, Morris-Stiff G. Next-generation sequencing in pancreatic cancer. Pancreas 2019;48:739-748.

2. Imaoka H, Sasaki M, Hashimoto Y, Watanabe K, Ikeda M. New era of endoscopic ultrasound-guided tissue acquisition: nextgeneration sequencing by endoscopic ultrasound-guided sampling for pancreatic cancer. J Clin Med 2019;8:1173.

3. Biankin AV, Waddell N, Kassahn KS, et al. Pancreatic cancer genomes reveal aberrations in axon guidance pathway genes. Nature 2012;491:399-405.

4. de Biase D, Visani M, Acquaviva G, et al. The role of next-generation sequencing in the cytologic diagnosis of pancreatic lesions. Arch Pathol Lab Med 2018;142:458-464.

5. Kameta E, Sugimori K, Kaneko T, et al. Diagnosis of pancreatic lesions collected by endoscopic ultrasound-guided fine-needle aspiration using next-generation sequencing. Oncol Lett 2016;12 3875-3881

6. de Biase D, Visani M, Baccarini P, et al. Next generation sequencing improves the accuracy of KRAS mutation analysis in endoscopic ultrasound fine needle aspiration pancreatic lesions. PLoS One 2014;9:e87651.

7. Larson BK, Tuli R, Jamil LH, Lo SK, Deng N, Hendifar AE. Utility of endoscopic ultrasound-guided biopsy for next-generation sequencing of pancreatic exocrine malignancies. Pancreas 2018;47: 990-995.

8. Elhanafi S, Mahmud N, Vergara N, et al. Comparison of endoscopic ultrasound tissue acquisition methods for genomic analysis of pancreatic cancer. J Gastroenterol Hepatol 2019;34:907-913.

9. Affolter KE, Schmidt RL, Matynia AP, Adler DG, Factor RE. Needle size has only a limited effect on outcomes in EUS-guided fine needle aspiration: a systematic review and meta-analysis. Dig Dis Sci 2013;58:1026-1034.

10. Park JK, Lee JH, Noh DH, et al. Factors of endoscopic ultrasoundguided tissue acquisition for successful next-generation sequencing in pancreatic ductal adenocarcinoma. Gut Liver 2020;14:387394. 